\title{
Long-term variation in the short-period binary system WR 46 (WN3p+?)
}

\author{
Pieter M. Veen ${ }^{1}$, Arnout M. van Genderen ${ }^{1}$, and Albert F. Jones ${ }^{2}$ \\ ${ }^{1}$ Leiden Observatory, PO Box 9513, NL-2300 RA Leiden, \\ the Netherlands \\ ${ }^{2}$ Carter Observatory, Wellington, New Zealand
}

\begin{abstract}
We studied the short-period $(P \simeq 6-8 \mathrm{hr})$ binary system WR 46 using simultaneous photometry and spectroscopy. We show that the long-term photometric variation is accompanied by changes in the equivalent width of the He II $\lambda 5411$ emission line and by an apparent period decrease on the rising branch. Furthermore, the brightening of the object is repeating.
\end{abstract}

\section{Exposition}

The object WR 46 (HD 104994, WN3p) is classified peculiar, because it is the only WN star showing strong Ovi lines at $3811 / 34 \AA$. On the basis of an ellipsoidal light-curve $(P=0.2824 \mathrm{~d}$, van Genderen et al. 1991) and a radial-velocity curve (Veen et al. $1995 ; P=0.31 \mathrm{~d}$, Niemela et al. 1995) the object is suggested to be a close binary. The alleged companion does not show any spectral features.

Observations by the Hipparcos satellite (Marchenko et al. 1998) were the first to show variability on a time-scale of years. Walraven photometry, taken partly simultaneously, confirms the rise in 1990 and 1991 (Fig. 1). The open symbols in the plot are mean values from several monitoring runs. Also indicated are the visual eye estimates $(\sigma \sim 0.1)$ by one of us $(\mathrm{AJ})$. The latter observations prove the system to vary on a time-scale of months in 1998, as confirmed by the CCD photometry. Fig. 1 (bottom) shows the long-term variation of the equivalent

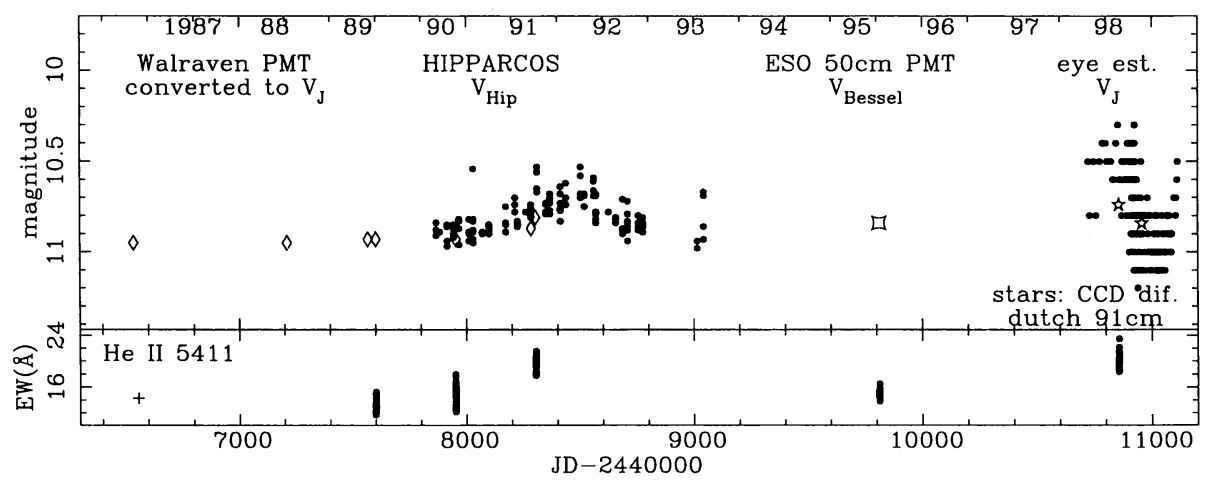

Figure 1. In phase long-term variability of photometry and $E W$. 


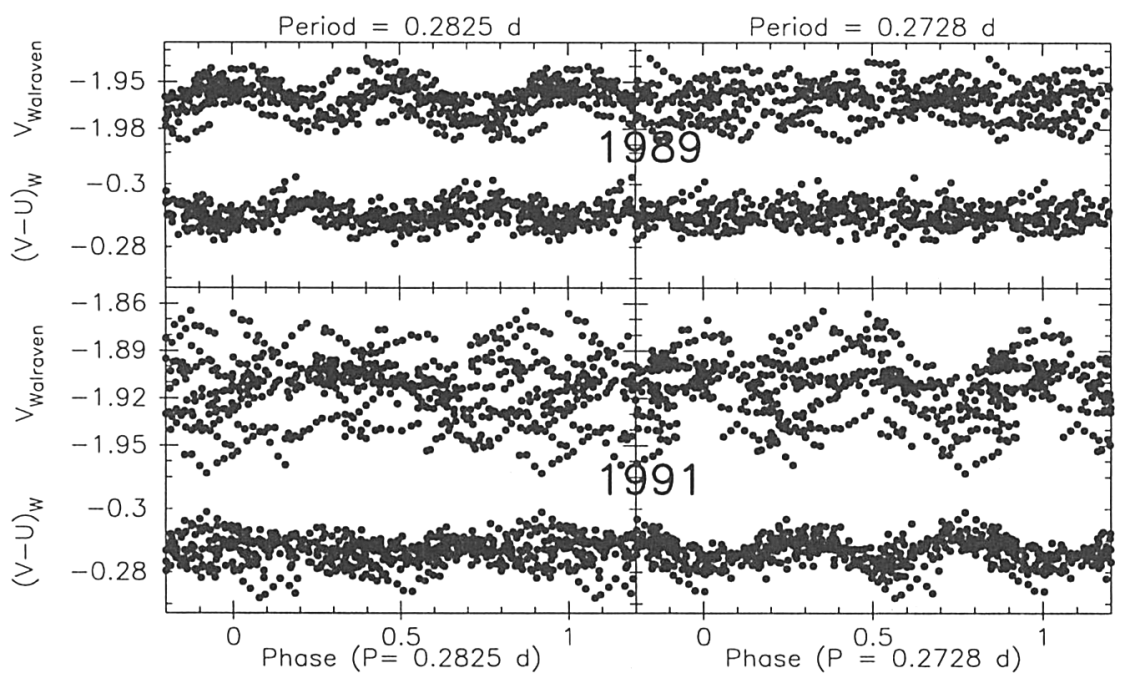

Figure 2. The period as determined in 1989 (left) and 1991 (right).

width of He II $\lambda 5411$ emission line during our monitoring runs using the ESO $1.5 \mathrm{~m}$ telescope. The datapoint in 1986 is measured from a spectrum taken at the $3.6 \mathrm{~m}$ ESO telescope using CASPEC. The $E W$ is in phase with the photometric variation in the top panel.

Also the period seems to change, seemingly shorter with increasing brightness. Fig. 2 presents light- and colour-curves from two data sets (log-intensity scale), 1989 (top) and 1991 (bottom), folded with $P=0.2825 \mathrm{~d}$ (left) and with $P=0.2728 \mathrm{~d}$ (right). In addition, the cycle-to-cycle differences are larger in 1991 relative to 1989 , suggesting that the continuum radiating layers were more unstable. The colour curves are helpful in identifying the period with the smallest scatter. A change of 0.0096 day $(14 \mathrm{~min})$, i.e., $\frac{\Delta P}{P}=0.03$ in two years, is extremely large for a binary system. Whether the unseen companion is truly spiralling inwards is doubtful. It is not ruled-out that effects of the unstable atmosphere played a trick on us.

Acknowledgments. We thank Werner Schmutz for the CASPEC spectra.

\section{References}

van Genderen, A.M., Verheijen, M.A.W., van Kampen, E., et al. 1991, in: K.A. van der Hucht \& B. Hidayat (eds.), Wolf-Rayet Stars and Interrelations with Other Massive Stars in Galaxies, Proc. IAU Symp. No. 143 (Dordrecht: Kluwer), p. 129 Marchenko, S.V., Moffat, A.F.J., van der Hucht, K.A., et al. 1998, A\&A 331, 1022 Niemela, V.S., Barbá, R.H., Shara, M. 1995, in: K.A. van der Hucht, K.A. \& P.M. Williams (eds), Wolf-Rayet stars: Binaries, Colliding Winds, Evolution, Proc. IAU Symp. No. 163, (Dordrecht: Kluwer), p. 245

Veen, P.M., Van Genderen, A.M., Verheijen, M.A.W., et al. 1995, in: ibid., p. 243 